\title{
Drug-Core Double Emulsions for Co-release of Active Ingredients
}

\author{
E. Dluska, A. Markowska-Radomska, A. Metera, and W. Tomaszewski
}

\begin{abstract}
The paper presents the results of the release process of two encapsulated drugs from double emulsions. Double emulsions have been proposed and discussed as a promising carriers of a few drugs for effective multidrug therapy. Double emulsions were formed by one step method in a Couette-Taylor flow (CTF) bioreactor. Emulsions of $\mathrm{O}_{1} / \mathrm{W} / \mathrm{O}_{2}$ type with different structures were obtained. Experimental release study involved the influence of process parameters in the CTF bioreactor (liquid flow rates, rotational frequency of inner cylinder of the bioreactor and its annular gap) on the release rates of two entrapped model active agents from internal droplets to external continuous phase of emulsions. Simultaneous release of active agents entrapped in $\mathrm{O}_{1} / \mathrm{W} / \mathrm{O}_{2}$ emulsions proceed as two steps process. In the first step the simultaneous release of both substances has been observed, whereas in the second step only remain substance having larger molecule. The results shown the influence of the preparation conditions on the kinetics of the release process.
\end{abstract}

Index Terms-Multiple emulsions, encapsulation, release process, bioreactor.

\section{INTRODUCTION}

Multiple emulsions are complex dispersed systems that contain at least three liquid phases: inner and membrane both dispersed phases and outer continuous phase (Fig. 1). The simplest structures of multiple emulsions are double emulsions $(\mathrm{O} / \mathrm{W} / \mathrm{O}$ or $\mathrm{W} / \mathrm{O} / \mathrm{W}$ type, $\mathrm{O}$ - oil phase, $\mathrm{W}$ - water phase).

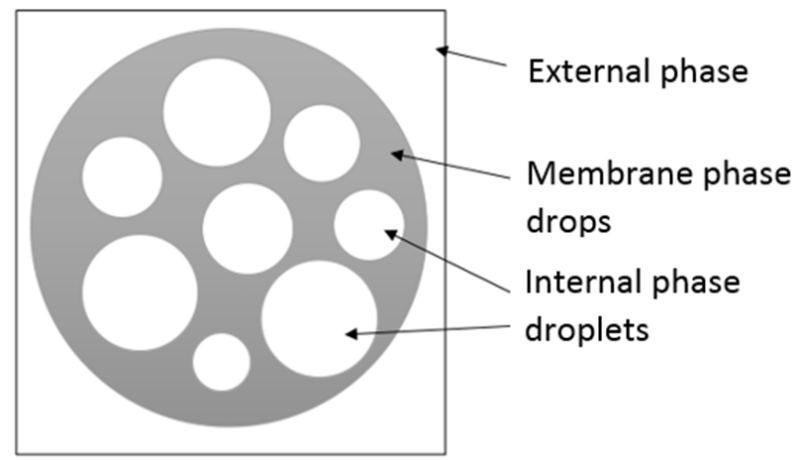

Fig. 1. The structure of double emulsions.

The complex structure of multiple emulsions are used as carrier systems to encapsulate and delivery lipophilic and

Manuscript received June 28, 2016; revised August 17, 2016. This work was supported by the National Science Centre - Poland under Grant Number 2014/13/B/ST8/04274.

E. Dluska, A. Markowska-Radomska, and A. Metera from Warsaw University of Technology, Faculty of Chemical and Process Engineering, 00-645 Warsaw, Warynskiego 1 (e-mail: e.dluska@ichip.pw.edu.pl, a.markowska@ichip.pw.edu.pl, a.metera@ichip.pw.edu.pl). hydrophilic substances (e.g drugs, food additives) and living cells [1]-[3]. The inner and outer phases of multiple emulsions are separated by membrane phase that protects encapsulated active agents from damaging phenomena during processing or storage. The membrane phase also influences and modify the rate of the processes occurring in the multiple emulsion based systems e.g. controlled release or extraction of active substances.

Multiple emulsions due to ability to control the release rate provide plasma concentration of active agents in the range of therapeutic window what is extremely important in therapies controlled and modified [4]. Modified release of a drug could be delayed, sustained, controlled, organ targeted or cell receptor specific release [5]. Special formulations of drugs could be drug delivery systems that provide modified release controlled by different mechanisms e.g. diffusion, osmosis, swelling and dissolution and degradation [6].

Research to provide formulation of drugs and functional food products to delivery more than one active agent involve systems for drug delivery, such as nanoparticles, liposomes, nanofibers and recently also multiple emulsions. Recently there has been an increasing interest in studying novel drugs formulation on simultaneously delivery of two or more drugs to achieve better therapeutic effects due to drugs interaction.

Multiple emulsions allow hydrophilic and hydrophobic substances or even three different active agents to be encapsulated and then released in control manner. As research show double emulsion could contain such as sodium lactate, spironolactone and chlorhexidine digluconate in W/O/W double emulsion [7]. Encapsulation and release of metronidazole with ornidazole in internal and external phases of W/O/W emulsion for vaginal delivery were investigated by Özer et al. [8].

The modern microencapsulation technology of bioactive substances is an important formulation strategy to multidrug therapies being involved into advanced drug delivery systems. Multidrug therapy was first proposed to the treatment of tuberculosis and is practice widely adopted against AIDS and cancer. Although in the present literature of drug delivery systems most publications focus on the encapsulation of larger molecules, e.g., peptides, proteins, and DNA/RNA for potential use as vaccines or as long-acting release (LAR) drug formulations, no precise data are available on co-release kinetics of co-encapsulated drugs.

The presented work aimed to study the effect of simultaneous encapsulation of two different model substances (drugs) in double emulsions formed in a Couette- Taylor Flow (CTF) bioreactor on their release kinetics and mechanisms. The results of co-release of model drugs were discussed to evaluate the possibility of modification of release process 
kinetics by incorporation of different substances within structure of double emulsion for multidrug therapies.

\section{DRUG-CORE DOUBle EMULSIONS FORMATION IN A COUETTE-TAYLOR FLOW BIOREACTOR FOR RELEASE EXPERIMENTS}

The purpose of the experiments was to obtain active multiple (double) emulsions with two different substances (drugs) by encapsulation and emulsification processes conducted in a Couette-Taylor flow bioreactor (CTF) for drug co-release kinetics study. It has been investigated the influence of hydrodynamic parameters in CTF bioreactor (rotational frequency of internal cylinder in bioreactor, flow rates) and initial concentration of drug that has been encapsulated with phenyl salicylate on multiple emulsion characteristics (size and drop size distribution, volume packing of internal droplets), encapsulation efficiency and rate of drugs release. The scope of the experimental study included kinetic stability and release mechanism of drugs from multiple emulsions.

\section{A. Materials and Composition of $\mathrm{O}_{1} / \mathrm{W} / \mathrm{O}_{2}$ Emulsion}

The study was conducted for two model active substances: phenyl salicylate (salol) as a base ingredient, and benzoic acid as the second ingredient (Fig. 2). Phenyl salicylate is used as an antiseptic agent in urinary tract and digestive tract infection. Benzoic acid is popular food and drugs preservatives.
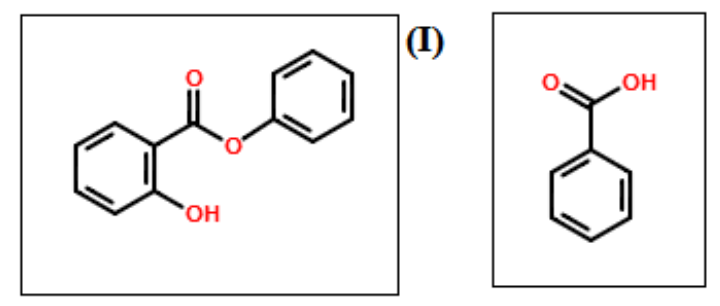

Fig. 2. The structural formulas of drugs encapsulated in multiple emulsions: I- phenyl salicylate (salol), II- benzoic acid.

The compositions of double emulsions (1), (2) and (3): $\left(\mathrm{O}_{1}\right)$ - the internal oil phase was liquid paraffin (Sigma) + salol 10 wt.\% (Sigma) for all emulsions + benzoic acid: $9.09 \cdot 10^{-3}$ wt.\% for emulsions (1); $2.95 \cdot 10^{-3} \mathrm{wt} . \%$ for emulsions (2) and $2.91 \cdot 10^{-3}$ wt.\% for emulsions (3) (Sigma); (W) - the membrane water phase was distilled water + gelatin for microbiology uses (pH=4.0-6.0, 240-270g Bloom) 15 wt.\% $($ Sigma $)+$ sucrose 5 wt.\% (Fluka) for all emulsions; $\left(\mathrm{O}_{2}\right)$ - the external oil phase was liquid paraffin (Sigma) for all emulsions.

\section{B. Method of Formation of $\mathrm{O}_{1} / W / \mathrm{O}_{2}$ Emulsion and Drugs Encapsulation}

The simultaneous processes of emulsification and encapsulation of two active substances (model drugs) in internal droplets (cores) of double emulsions were carried out as a one-step process in a continuous Couette-Taylor flow (CTF) biocontactor/bioreactor (Fig. 3). The drug-core double emulsions were formed at different hydrodynamic conditions (the rotational frequency of the inner cylinder: 1400-1850 $\mathrm{rpm}$, the size of annular gap between the coaxial cylinders: 1.5 $\mathrm{mm}$, the volumetric flow rates of oils, and water phases: $\mathrm{V}_{\mathrm{O} 1}=30-50 \mathrm{~cm}^{3} / \mathrm{min}, \mathrm{V}_{\mathrm{W}}=10-15 \mathrm{~cm}^{3} / \mathrm{min}, \mathrm{V}_{\mathrm{O} 2}=100 \mathrm{~cm}^{3} / \mathrm{min}$ ) for different initial concentrations of active ingredients (benzoic acid $2.95 \cdot 10^{-3}-9.09 \cdot 10^{-3}$ wt.\%, salol: 10 wt. \%). A detailed description of the double/multiple emulsion preparation via one step method in a CTF contactor can be found in our previous papers [9], [10].

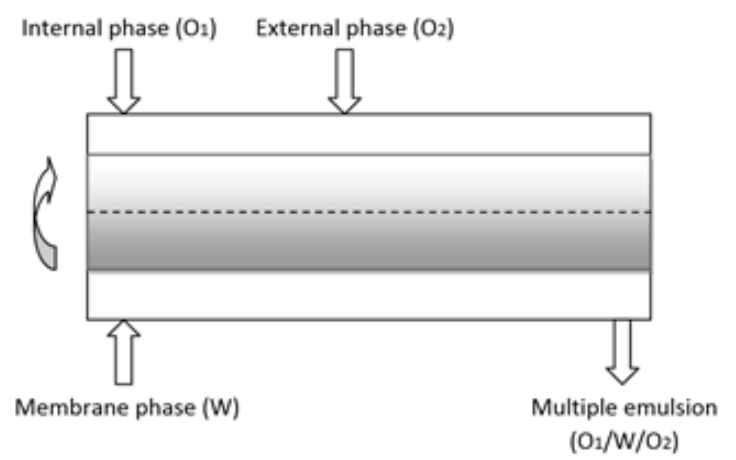

Fig. 3. A Couette-Taylor flow biocontactor for forming multiple emulsions.

\section{Characterisation of $\mathrm{O}_{1} / \mathrm{W} / \mathrm{O}_{2}$ Emulsion with Active Substances}

In this study, the drop diameters of the internal phase (d) and the membrane phase (D) were determined based on microscopic observation by using an optical microscope (BX-60, Olympus) connected to a digital color camera (SC50, Olympus) and image analysis software Image Pro Plus 4.5 (Media Cybernetics). At least 600 drops of membrane phase and 1000 droplets of the internal phase of $\mathrm{O}_{1} / \mathrm{W} / \mathrm{O}_{2}$ emulsions were counted for measurements of the drop size for each emulsion sample. Based on the measured drop sizes, the Sauter mean diameter of internal $\left(\mathrm{d}_{32}\right)$ and membrane phase drops $\left(D_{32}\right)$ have been calculated.

The volume packing fraction $(\phi)$ for studied multiple emulsions was calculated as the ratio of the volume of internal droplets to the volume of the membrane phase drops.

The measurements of double emulsions stability were carried out based on microscopic observation of emulsion over time. There were no changes of the drops morphology of inner and membrane phase of double emulsions stored at $37^{\circ} \mathrm{C}$ during 7 days observation.

Encapsulation efficiency (EE) of active substances (salol, benzoic acid) was calculated according to the difference between the amount of substance added to the inlet stream of internal phase to CTF contactor and the unentrapped amount of substance measured in the external oil phase after emulsification process. Samples of external paraffin phase were taken immediately after preparation from the multiple emulsions using hydrophobic syringe filters (pore size- 0.45 $\mu \mathrm{m}$; Milipore).

The concentration of active agent was measured based on standard method: (i) HPLC (high performance liquid chromatography) for phenyl salicylate and (ii) GC (gas chromatography) for benzoic acid. The samples were extracted in ultrasonic bath at $30^{\circ} \mathrm{C}$ during $15-30 \mathrm{~min}$. Then were extracted in $5 \mathrm{~cm}^{3}$ graduated flask, containing acetonitrile. For benzoic acid analysis also the water was added to the mark. Before measurements extracts were filtered using $0.45 \mu \mathrm{m}$ PTFE disposable filters (Milipore). 
HPLC quantitative analysis of benzoic acid was carried out using Agilent 1260 Infinity liquid chromatograph equipped with UV-DAD detector. The mobile phase was acetonitrile/water $50 / 50$ vol., both solvents were acidified with $\mathrm{H}_{3} \mathrm{PO}_{4}$, to get concentration of $0.02 \mathrm{M}$.

The flow rate was $1 \mathrm{~cm}^{3} / \mathrm{min}$, detection at $241 \mathrm{~nm}$ was applied and injection $25 \mathrm{~mm}^{3}$. The analysis was carried out using Supelcosil ABZ+ column 150x4.6 mm with precolumn $10 \times 4.6 \mathrm{~mm}$ at $60^{\circ} \mathrm{C}$. HPLC grade solvents were used. The quantitative analyses of salol were performed using GCMS system equipped with gas chromatograph GC 7890A coupled with mass detector VL MSD 5975C, both from Agilent.

A capillary column HP-1701 $30 \mathrm{~m}$ x $250 \mu \mathrm{m}$ x $0.25 \mu \mathrm{m}$ was used, with helium as a carrier at $1 \mathrm{ml} / \mathrm{min}$. Sample volume was $0.5 \mathrm{~mm}^{3}$ at split value of $1: 10$ and injector temperature $200^{\circ} \mathrm{C}$. For preparing of samples HPLC grade solvents were used. The analysis was performed in TIC mode at $\mathrm{m} / \mathrm{z}$ 10-700. Each experiment was performed in triplicate.

\section{In Vitro Co-release Experimental Study}

The release study concerned co-release of two entrapped model active agents (salol and benzoic acid) from the internal paraffin droplets to the external paraffin phase of double emulsions $\mathrm{O}_{1} / \mathrm{W} / \mathrm{O}_{2}$. Release studies of salol and benzoic acid were performed for three sets of emulsions obtained in CTF bioreactor under $1802 \mathrm{rpm}$ (emulsions 1 and 3) and $1622 \mathrm{rpm}$ (emulsion 2). The investigated emulsions differed in their internal structures (drop size and volume packing fraction) and the encapsulation efficiency of active substances.

The release experiment were performed at $37^{\circ} \mathrm{C}$ in a standard glass stirrer tank with a diameter of $10 \mathrm{~cm}$ and volume $0.976 \mathrm{dm}^{3}$, for rotational frequency of stirrer $250 \mathrm{rpm}$ (Fig. 4). The tank was equipped with a four plane blade turbine impeller with the blades set an angle of $45^{\circ}$, diameter $5 \mathrm{~cm}$; blades width $0.5 \mathrm{~cm}$.

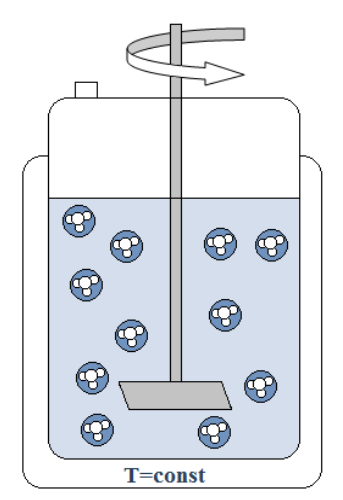

Fig. 4. Experimental set-up for testing the release of drugs encapsulated in double emulsions.

In order to determine the release rates of salol and benzoic acid the samples of the external phase of double emulsions were taken at specified intervals time similarly to the measurements of encapsulation efficiency (EE).

The concentration of salol was measured by HPLC and for benzoic acid by GC (see Chapter II.C.) Each experiment was repeated three times. On the base of the changes in concentrations of salol and benzoic acid over the time of experiments the cumulative mass fraction of released two examined model active agents from $\mathrm{O}_{1} / \mathrm{W} / \mathrm{O}_{2}$ double emulsion were determined.

\section{RESULTS AND DISCUSSION}

The drug- core double emulsions $\mathrm{O}_{1} / \mathrm{W} / \mathrm{O}_{2}$ formed by one step method in a Couette-Taylor flow (CTF) bioreactor differed in their internal structure depending on hydrodynamic conditions in the bioreactor. Figs. 5a and 5 b show examples of microscopic image and drop size distribution of obtained emulsions (1).

The parameters characterising internal structures of emulsions like drops sizes, volume packing of internal droplets in the membrane phase drops and encapsulation efficiency of each ingredients (salol and benzoic acid) are presented in legends of Figs. 6 and 7.
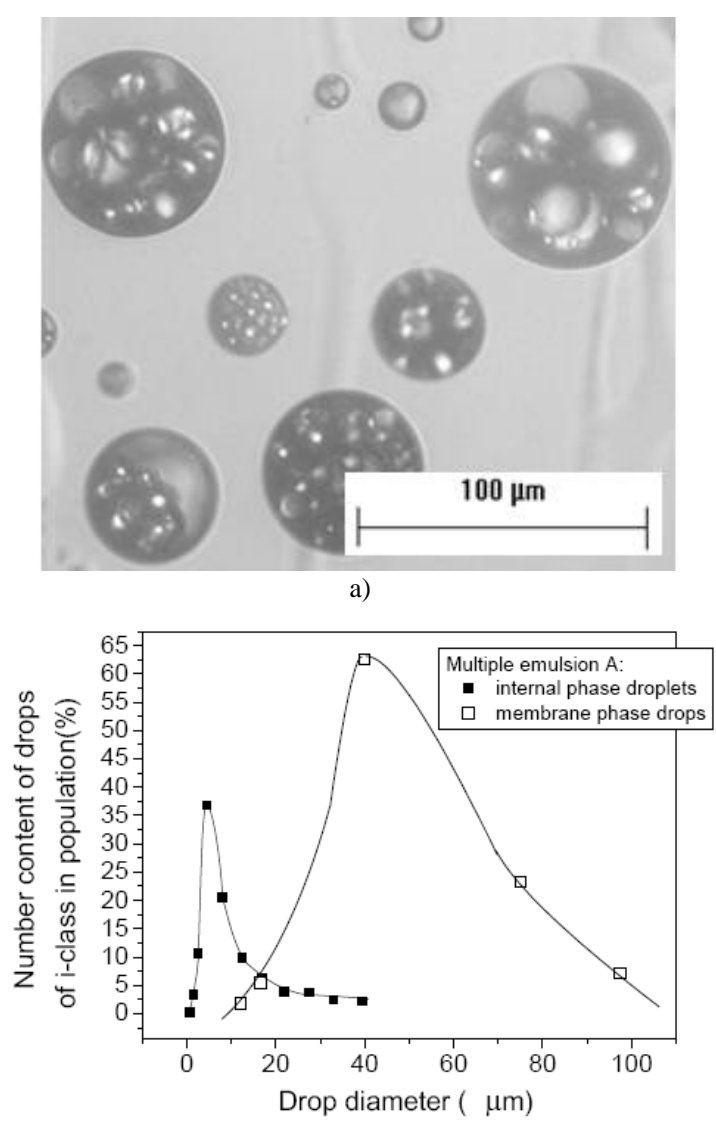

b)

Fig. 5. The $\mathrm{O}_{1} / \mathrm{W} / \mathrm{O}_{2}$ double emulsions (1): a) microscopic image, b) size distribution of internal and membrane drops in population of $\mathrm{O}_{1} / \mathrm{W} / \mathrm{O}_{2}$ emulsions.

The results of co-release of two model drugs are presented in form of cumulative mass of each ingredient released over time in Figs. 6-8. The release of both active substances from double emulsions prepared under different hydrodynamic conditions in the CTF bioreactor occurred according to diffusion mechanism, which has been verified during investigation of release process on the basis of microscopic images analysis.

The profiles of co-release of phenyl salicylate (salol) and benzoic acid entrapped in $\mathrm{O}_{1} / \mathrm{W} / \mathrm{O}_{2}$ emulsions demonstrated two-stage kinetics for salol (Fig.6) and one-stage kinetics for benzoic acid (Fig. 7). In the first stage of salol release the simultaneous release of both substances has been observed, 
whereas in the second step only remain substance having larger molecule (salol). The two-stage release profiles of basic substance i.e. salol ( showed in Fig. 6) resulted from the addition of second substance (benzoic acid) which also reduced time needed to completely release of encapsulated phenyl salicylate. The longer time necessary for completely release of phenyl salicylate was observed for those emulsions which have been prepared under higher rotational frequency of internal cylinder in the CTF contactor. The results confirmed the effect of hydrodynamic conditions in the CTF bioreactor on the release rates which influenced the structures of obtained emulsions and thus release rates (Fig. 8). As expected an active substance has been faster released from the emulsions (2) characterised by smaller encapsulation efficiency (EE) and drop volume packing $(\phi)$ compared to emulsions (3).

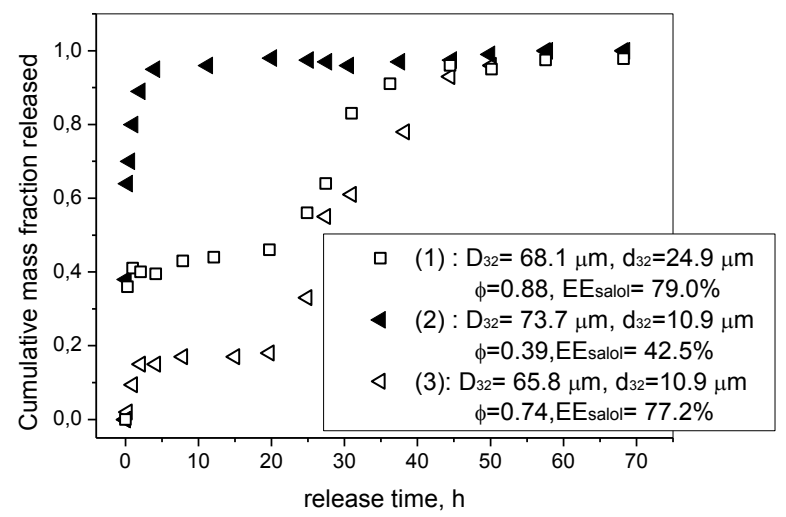

Fig. 6. The experimental release profiles of salol from double emulsions 1, 2 and 3 under mixing of release environment: $250 \mathrm{rpm}$.

The longer time necessary for completely release of phenyl salicylate was observed for those emulsions which have been prepared under higher rotational frequency of internal cylinder in the CTF contactor.

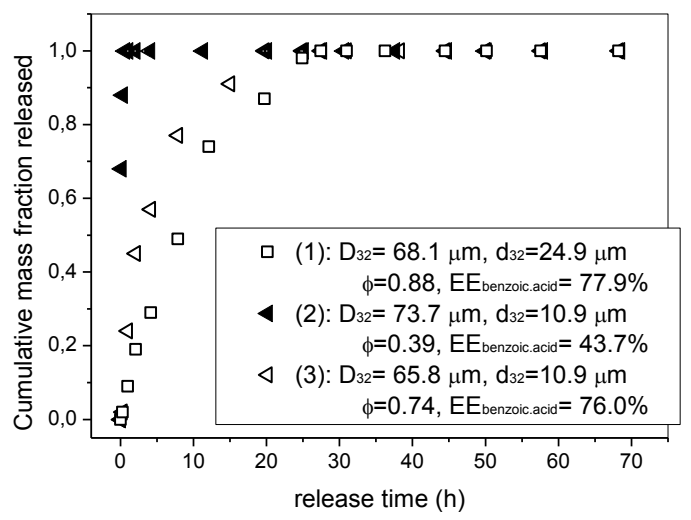

Fig. 7. The experimental release profiles of benzoic acid from double emulsions (1), (2) and (3) under mixing of release environment: $250 \mathrm{rpm}$.

The results confirmed the effect of hydrodynamic conditions in the CTF bioreactor on the release rates which is associated with different internal structures of emulsions obtained at different flow field conditions (Fig. 8). As expected faster release of an active substance (benzoic acid) has been observed from the emulsions (2) characterised by smaller encapsulation efficiency (EE) and drop volume packing $(\phi)$ compared to emulsions (3).

Former studies on formation double emulsions $\mathrm{O}_{1} / \mathrm{W} / \mathrm{O}_{2}$ in a Couette -Taylor flow contactor contained phenyl salicylate (salol) as one encapsulated active agent shown one step release profiles. The comparison of release profiles of drugs from double emulsions with one and two encapsulated drugs is presented in Fig.9.

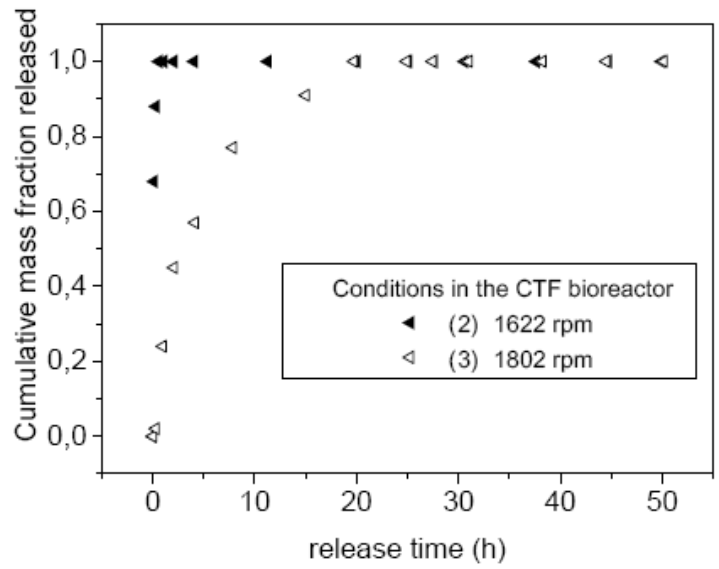

Fig. 8. The influence of rotational frequency of the inner cylinder in the CTF bioreactor (formation conditions) on the release rates of benzoic acid from double emulsions (2) and (3).

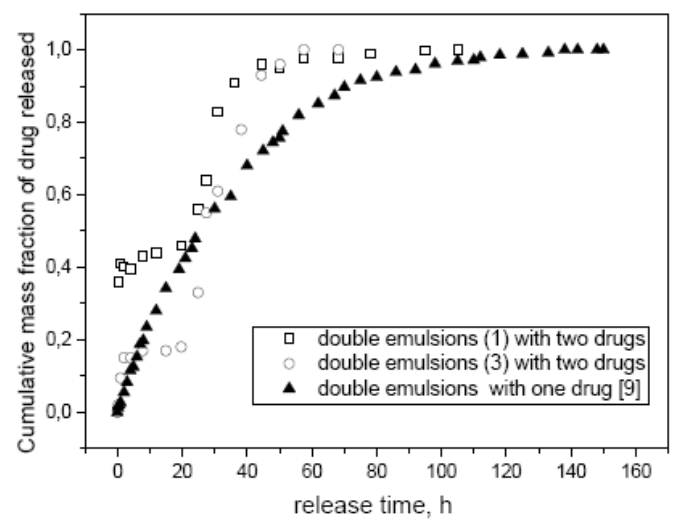

Fig. 9. The comparison of drug release process from double emulsions $\mathrm{O}_{1} / \mathrm{W} / \mathrm{O}_{2}$ with one and two encapsulated drugs.

Comparison of structure of multiple emulsions with salol as a one model drug and emulsions with simultaneously encapsulated salol and benzoic acid shown the significant influence of second encapsulated active agent (benzoic acid) on characteristics of multiple emulsions, release rates and type of release profiles. The characteristics of double emulsions with two drugs shown that co-encapsulation influenced the reduction of Sauter mean diameter of inner droplets and membrane phase drops of multiple emulsions, encapsulation efficiency of salol and stability of multiple emulsions. Addition of second model drug (benzoic acid) into inner phase forming internal droplets of $\mathrm{O}_{1} / \mathrm{W} / \mathrm{O}_{2}$ in a process of encapsulation and emulsification caused modification of basic drug (salol) release from one stage to a two- stage release kinetics and also reduced time necessary to its complete release.

The proposed method of multiple emulsions formation and encapsulation of drugs in the CTF bioreactor have a great potential as a simple, effective, less time consuming method to produce advanced formulation of drugs, comparing to the conventional methods (two-step mechanical mixing). Due to 
the fact that emulsification and encapsulation processes are conducted in one apparatus (one-step process) this method provides safety of work, especially with active agents for medical purpose e.g. anticancer drugs. Former studies show that the physical properties and structure of obtained products are strictly associated with values of operating conditions of the process conducting in a CTF bioreactor.

Moreover, this paper presents the composition of liquid solutions and active substances introduced to the CTF bioreactor that could significantly influence the properties of formed multiple emulsions and modify release process of active substances. Two-stage release profiles obtained for model active agents encapsulated in the inner droplets of $\mathrm{O}_{1} / \mathrm{W} / \mathrm{O}_{2}$ double emulsions showed that through programmable addition of appropriate drugs and their amount more complex modified delivery system could be successfully created.

Literature overview shown that advanced drug formulations provided modified release of two different active agents potentially could be useful as drug delivery systems in multidrug or targeted therapies e.g. to deliver a few different anticancer drugs or substances like protein or sh-DNA.

\section{SUMMARY}

The results of co-release a few active substances into double emulsions confirmed that encapsulation of biocompatible substances, which do not react, may provide a means to modify the release kinetics involving rate and shape of drug release profiles and influence the release mechanisms.

The modification of release process of active ingredients from double emulsions can be controlled by hydrodynamic parameters in a Couette-Taylor flow bioreactor, which influence sizes of internal droplets and drops forming a liquid-permeable membrane, separating the internal droplets from the external environment, as well as fraction of volume packing. In this study a desirable modified types of release profiles have been achieved by adding a few active ingredients into internal droplets. It is also possible adding ingredients into membrane phase drops of double emulsions for modifying release rates and shape of release profiles.

Research on simultaneous encapsulation of a few substances into double emulsions and co-release that have been made until now allowed developing new potential applications of multiple emulsions, as a systems for controlled drugs release in multidrug therapies used when conventional treatment is not sufficiently effective.

\section{SYMBOLS USED}

$D_{32}, d_{32}$ - the Sauter mean diameter of membrane and internal phase drops of double emulsions, $\mu \mathrm{m}$

EE - encapsulation efficiency, \%

$\phi$ - volume packing fraction of internal droplets in the membrane phase drops of double emulsions.

\section{REFERENCES}

[1] A. Aserin, Multiple Emulsions: Technology and Applications, Hoboken, NJ: J. Wiley \& Sons, 2008.

[2] M. L. Shively, "Multiple emulsions for the delivery of proteins," in Protein Delivery, L. M. Sanders, R. W. Hendren, Eds. New York: Springer US, 2002, pp. 199-211.

[3] R. Kumar, M. S. Kumar, and N. Mahdevan, "Multiple emulsions: A review," Int J Recent Adv Pharm Res, vol. 2, no. 1, pp. 9-19, 2012

[4] C. G. Wilson, "The need for drugs and drug delivery systems," in Fundamentals and Applications of Controlled Release Drug Delivery. Advances in Delivery Science and Technology, J. Siepmann, R. A. Siegel, M. J. Rathbon, Eds. New York: Springer US, 2012, pp. 3-18.

[5] J. P. Remington and P. Beringer, Remington: The Science and Practice of Pharmacy, 21nd Edition, D. B. Troy, Ed., Philadelphia: Lippincott Williams \& Wilkins, 2006, pp. 939-940.

[6] P. L. Lam and R. Gambari, "Advanced progress of microencapsulation technologies: In vivo and in vitro models for studying oral and transdermal drug deliveries," J Control Release, vol. 178, pp. 25-45, 2014.

[7] S. Raynal, J. L. Grossiord, M. Seiller, and D. Clausse, "A topical W/O/W multiple emulsion containing several active substances: Formulation, characterization and study of release," J Control Release, vol. 26, no. 2, pp. 129-140, 1993.

[8] Ö. Özer, M. Özyazici, M. Tedajo, M. S. Taner, and K. Köseoglu, "W/O/W multiple emulsions containing nitroimidazole derivates for vaginal delivery," Drug Deliv, vol. 14, no. 3, pp. 139-145, 2007.

[9] E. Dluska and A. Markowska, "One-step preparation method of multiple emulsions entrapping reactive agent in the liquid-liquid Couette-Taylor flow," Chem Eng Proces, vol. 48, no. 1, pp. 438-445, 2009.

[10] E. Dluska and A. Markowska-Radomska, "Regimes of multiple emulsions of $\mathrm{W} 1 / \mathrm{O} / \mathrm{W} 2$ and $\mathrm{O} 1 / \mathrm{W} / \mathrm{O} 2$ type in the continuous Couette-Taylor flow contactor," Chem Eng Technol, vol. 33, no. 1, pp. 113-120, 2010.

Ewa Dluska is Associate Professor at the Warsaw University of Technology. He got the PhD in 1997 and DSC in 2012 in chemical engineering from the Warsaw University of Technology. He is Research fellowship at the University of Oxford in the Institute of Biomedical Engineering in encapsulation of stem cells in 2013. His research interests are encapsulation of drugs/living cells, release processes, developing of emulsions with bioactive agents in a Couette-Taylor Flow bioreactor for drug delivery systems; Modelling of $\mathrm{dmg}$ release process from emulsions and micro/nanoparticles; Separation process in environmental protection: removal of organic compounds and heavy metals using emulsion liquid membrane (ELM), modelling of the mass transfer and experimental investigations of process efficiency, etc. 